\title{
A multiscale approach of fatigue and shakedown for notched structures
}

\author{
G. Bertolino $^{\text {a }}$, A. Constantinescu ${ }^{\mathrm{a}, *}$, M. Ferjani ${ }^{\mathrm{a}}$, P. Treiber ${ }^{\mathrm{b}}$ \\ a Laboratoire de Mécanique des Solides - CNRS UMR 7649, Ecole Polytechnique, 91198 Palaiseau, France \\ ${ }^{\mathrm{b}}$ Institut für Materialprüfung - Universität Stuttgart, Germany
}

\begin{abstract}
The aim of this paper is to analyse the fatigue phenomena in the presence of stress gradients. It is well-known that most fatigue criteria fail to predict the lifetime of components in the presence of high stress concentrations or stress gradients, as it is the case in the neighbourhood of cracks, holes notches and encountered for example in riveted or threaded structures. Proposed is a numerical approach in the framework of the high cycle fatigue domain in order to give a qualitative answer. The work starts from the numerical computation of macroscopic loading corresponding to some fatigue experiments on specimens with an inclusion of metallic grains embedded in a macroscopic matrix. The computed fields are then analysed in terms of the HCF (high cycle fatigue) criterion [1], which is based on the estimation of the shakedown limit at the grain scale. The infinite lifetime prediction is based on the assumption that fatigue occurs if at least one grain fails, i.e. reaches plastic shakedown. The predictions at mesoscopic and macroscopic scales are close if the macroscopic stress distribution is homogeneous. However in the case of the stress gradient, lifetime predicted at the macroscopic scale is underestimated when compared to the predictions made at the mesoscopic scale. Another result is that the gap between microscopic and macroscopic predictions obtained from these numerical computations can roughly be estimated by a diminution of stress of the same order of magnitude as found in the experiments and phenomenological observations.
\end{abstract}

Keywords: Stress gradient; High cycle fatigue; Dang Van criterion; Polycrystalline plasticity

\section{Introduction}

A well-known fact in the engineering design of structures is that most fatigue criteria fail to predict correctly the lifetime of components in the presence of high stress concentrations or stress gradients.

\footnotetext{
* Corresponding author. Tel.: +3316933 33 30; fax: +33169 333026.

E-mail address: andrei.constantinescu@lms.polytechnique.fr (A. Constantinescu).
}

This is the case in the neighbourhood of cracks, holes notches, ... as encountered for example in riveted or threaded structures. More precisely, it can be stated that the local fatigue criteria based on the hot spot method, i.e. defining the most critical point in a structure such as the criterion in [1,2], generally underestimate the fatigue lifetime. The critical point of a structure in the neighbourhood of a geometrical stress concentration as angles, notches, cracks, etc., stems from the elastic solution of loading problem and the applied fatigue 
criterion. The problem addressed in this paper is an analysis of the physical phenomena at the smaller scales contributing to fatigue in order to give a qualitative explanation of this apparent contradiction.

Let us first mention that various phenomenological methods have been developed to overcome this difficulty. The concept of a "critical distance" [3,4] adapts the fatigue criterion by applying it at a given distance ahead of the notch tip. This distance is commonly of the order of the dimension of the metal grains. An application of this method to cylindrical notched specimen made of Ti6Al4V alloy is given in [3]. A similar concept, using a volumetric approach to compute an average stress taking place in a fatigue process zone, instead of the critical distance, has been proposed for example in $[5,6]$.

Other recent discussions at the macroscopic level of the influence of the stress gradient in bending fatigue experiments have been presented in $[7,8]$. The difficulties in applying fatigue criteria in the neighbourhood of stress concentrations arising in fretting fatigue problems is discussed in [9].

As a consequence of this short review of the models and discussions of lifetime predictions in the presence of stress gradient, one can finally conclude that the common practice is to include the stress gradient effect in fatigue analysis by introducing a notch factor or a critical distance parameter. This effectively corresponds to a lowering of the stress level and can be interpreted as the application of the fatigue criterion over a small averaging volume, rather than at the mathematical maximum point (hot spot method). This phenomenological approach provides acceptable results from the practical point of view. However a satisfactory link between the methodology and the underlying physical phenomena is still an open problem.

This paper proposes a numerical approach in the framework of the high cycle fatigue domain in order to give a first qualitative answer to this difficult question. The work starts from the numerical computation of macroscopic loading corresponding to some fatigue experiments on specimens with an inclusion of metallic grains embedded in a macroscopic matrix. As such the scale difference between the macroscopic level of the fatigue loading and the mesoscopic scale of the grains is gapped and as a consequence the apparition of shakedown at the grain scale can be observed and understood. If the fatigue analysis uses the criterion in [1] that is based on the estimation of the shakedown limit at the grain scale, then estimate can be made from the mechanical fields computed in the different grains if grain failure and therefore fatigue crack initiation are a possible outcome or not. The results present the distribution of mesoscopic pressure versus mesoscopic shear computed for the family of metallic grains considered and the permit to estimate the fatigue criterion [2] in each grain. Recall that the criterion in [2] is based on the assumption that fatigue occurs if at least one fails, i.e. reaches plastic shakedown. Therefore now it is possible to compare the fatigue predictions for the complete set of grains with the fatigue prediction by applying directly the criterion [2] at the macroscopic scale. One can easily remark that predictions at mesoscopic and macroscopic scales are close if the macroscopic stress distribution is homogeneous. However in the case of the stress gradient, fatigue occurs earlier if only the macroscopic scale is considered. Another result is that the gap between mesoscopic and macroscopic predictions can roughly be estimated to a diminution of stress of the same order of magnitude as in the phenomenological approached cited previously.

The paper starts with the presentation of the material model and of the simulated bending test in the second section. The next section recalls the basic assumptions underlying the mesoscopic and macroscopic fatigue criterion [1]. The forth section discusses the results of the computations in terms of fatigue predictions. The last section presents the conclusions of the work and opens some further research perspectives.

\section{Shakedown theory and Dang Van HCF}

Let us consider a structure under a mechanical loading. Its fatigue lifetime will be determined from the mechanical fields: elastic and plastic strains, stresses, etc. computed over a cycle. The underlying hypothesis is that the mechanical response of the structure is stabilized, i.e. that the fields will evolve under closed loops.

Fatigue phenomena can be characterized at three scales:

- the microscopic scale of dislocations, which are the underlying elements of persistent slip bands and elastoplastic strains;

- the mesoscopic scale of grains, where fatigue and damage phenomena are concentrated either at the grain boundary or in the interior; 
- the macroscopic scale of the structure, at which loads are applied and industrials design is performed.

An inspection of the three scales during a cyclic loading would conduct to different observations determined by the fatigue regime (see Fig. 1):

(i) In the high cycle fatigue regime ( $\mathrm{HCF})$, consider two fatigue domains corresponding to finite and infinite lifetime. Physical observations at the macroscopic scale show that structures are macroscopically in an elastic shakedown state. At the mesoscopic scale of the grains, it is now commonly accepted that elastic shakedown occurs only for the infinite lifetime. The case of a finite lifetime can be associated with a grain oriented in such a way with respect to the loading, that it can only not reach an elastic shakedown state. It will therefore be in plastic shakedown or ratcheting state which will conduct to failure after a finite number of cycles. The stress concentration due to this mesoscopic failure will therefore equally conduct a macroscopic crack initiation associated with failure.

(ii) In the low cycle fatigue regime (LCF), physical observation at both macroscopic and mesoscopic scales show extensive existence of plastic strains. Moreover homogenisation theory shows that strains and stresses at the two scales tend to be closer to each other with increasing plastic strain. This can be translated saying that the higher the applied load, the closer the mesoscopic and the macroscopic scales will behave.

In this work consider only the high cycle fatigue domain as defined in (i) and focus essentially on infi-

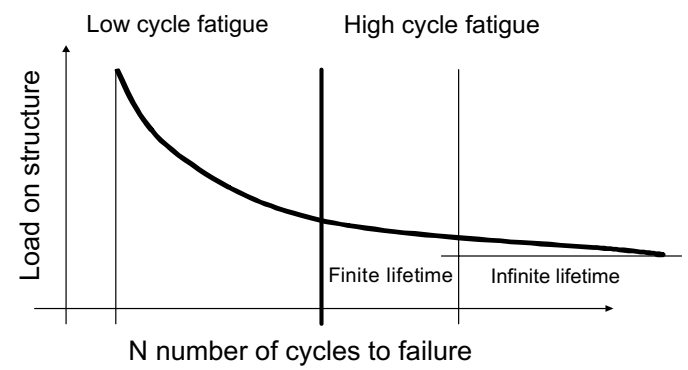

Fig. 1. Description of the low and high cycle fatigue domains in a Woehler diagram. nite lifetime. Recall the main assumptions of the fatigue criterion [2] as presented in [1]. As only an isolated grain will eventually be submitted to plastic shakedown, this problem is reduced to the analysis of an elastoplastic inclusion in an infinite elastic matrix and perform the passage between the macroscopic and mesoscopic fields using standard homogenisation theory. The homogenisation in the problem (2) that applies the hypothesis [3], i.e. equality of mesoscopic $\varepsilon$ and macroscopic strains $E$ :

$\varepsilon=E$

Under the assumption of equality of the macroscopic and mesoscopic moduli, one obtains after a short computation the following equality of macroscopic and mesoscopic stresses, $\Sigma$ and $\sigma$, respectively:

$\sigma=\Sigma-2 \mu \varepsilon^{p}=\Sigma+\rho^{*}$

where $\mu$ is the shear modulus and $\rho^{*}$ should be interpreted as a mesoscopic residual stress field. The knowledge of the mesoscopic stress tensor permits now to compute the mesoscopic shear stress $\tau(t)$ and the hydrostatic pressure $p(t)$.

The criterion in [2] states that the lifetime is infinite if elastic shakedown is reached at the macroscopic and the mesoscopic scale. This is equivalent to the statement, that the mesoscopic stress tensor satisfies the following inequality:

$\max [\tau(t)+a p(t)] \leqslant b$

where the coefficients $a$ and $b$ are defined from experiments as

$a=\frac{t_{\infty}-f_{\infty} / 2}{f_{\infty} / 3}, \quad b=t_{\infty}$

The $a$ and $b$ coefficients are deduced from two fatigue tests giving the fatigue limit $t_{\infty}$ in alternate torsion and $f_{\infty}$ in alternate flexion.

A common way to represent the criterion in [2] is to display the stress path in a mesoscopic shear $\tau(t)$ versus hydrostatic pressure $p(t)$ diagram as schematically drawn in Fig. 2. The line defined by the criterion is the frontier between infinite lifetime and fatigue, i.e. if a load path crosses the line, fatigue will occur.

From a practical point of view, one disposes actually of different algorithms which permit to automatically compute the mesoscopic shear stress $\tau(t)$ and the hydrostatic pressure $p(t)$ from the stress 


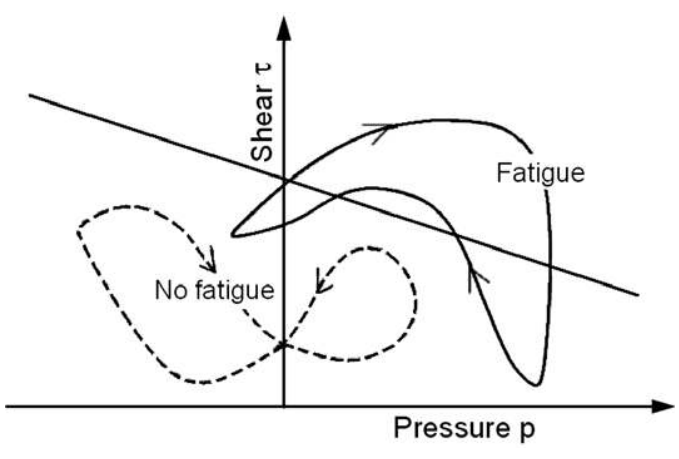

Fig. 2. Schematic view of the Dang Van criterion in the $\{\tau, P\}$ space.

results over a complete cycle of a finite element computation.

\section{Material and bending simulation}

\subsection{Model geometry}

The numerical model used in the present work consists of a domain that contains a number of grains which is surrounded by a homogeneous matrix. The domain containing the grains is the integral part of the fatigue analysis, whereas the homogeneous matrix allows the application of the loadings.

The grain domain shown in Fig. 3 includes 156 grains and has a rectangular geometry with side lengths of $147 \mu \mathrm{m}$ and $133 \mu \mathrm{m}$. The grain distribution was microscopically observed from an austenitic steel with an average grain size of about $10 \mu \mathrm{m}$.

The discussed austenitic steel consists of a facecentred cubic (FCC) crystal structure. The lattice

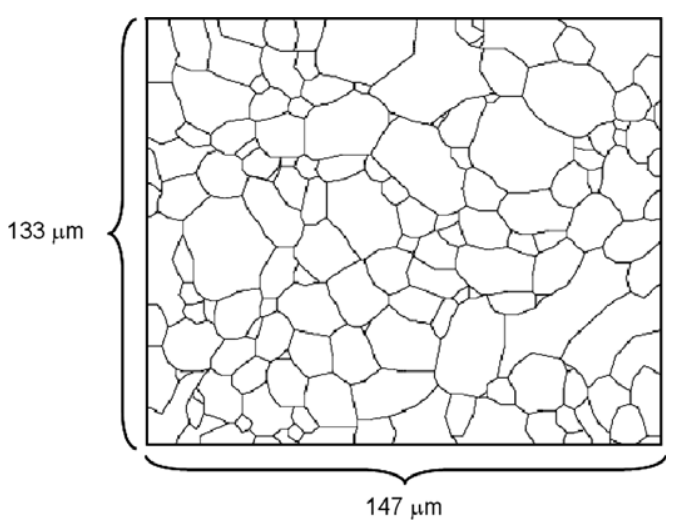

Fig. 3. Description of the micro-structural geometry of the grain inclusion. orientation has been observed with Orientation Imaging Microscopy using Electron Back Scattering Diffraction (EBSD). The individual grains were then meshed in two dimensions using linear three nodded elements.

\subsection{Materials properties}

The model used in the simulation consists of two different material properties which are applied to the grain containing domain and the homogeneous matrix. The homogeneous matrix was modelled with a simple linear elastic material law without plastic deformation. A more complex model is needed to describe the mechanical behaviour of the grains. The fatigue properties of a material are linked with grain plasticity and thus with the crystal structure. Therefore, it is necessary to consider the lattice structure of the material and to know the lattice orientation of all the grains.

From the macroscopic point of view, one can observe plastic deformation in a metal if the equivalent stress exceeds the yield limit $\sigma_{\mathrm{y}}$ of the material. On the microscopic scale, this plastic yielding is explained by the movement of dislocations in the crystal. This movement is induced by a force that acts on a slip plane in the direction of the Burger's vector (slip direction). These slip planes and slip directions depend on the lattice orientation and the direction of external forces. It is a well-known fact that the resulting shear stress and not the normal stress induces the movement of dislocations in the crystal and causes the plastic yielding. The resulting shear stress $\tau$ caused by a normal stress $\sigma$ that acts on a slip system can be calculated with the Schmid law given in the relation below [10-12]

$\tau=\sigma \cos \kappa \cos \lambda=\sigma S$

where $\kappa$ and $\lambda$ are the angles between the normal stress direction and the normal of the slip plane and slip direction, respectively. And $S$ is defined as the Schmid factor.

The shear force acting on a dislocation depends on the orientation of the slip system relative to the direction of the external normal stress. Each slip system has a corresponding Schmid factor. Considering an external normal stress, the slip system with the maximum Schmid factor is subjected to the highest shear stress. Local plastic yielding will occur if the shear stress $\tau$ in a slip system exceeds a threshold values $\tau_{0}$ and induce the movement of dislocations in this slip system. This threshold value is a 
Table 1

Slip planes and directions in a FCC crystal

\begin{tabular}{|c|c|}
\hline Slip plane & Slip directions \\
\hline (111) & {$[1-10],[-101],\left[\begin{array}{lll}0 & 1 & -1\end{array}\right]$} \\
\hline$(1-11)$ & {$[0-1-1],\left[\begin{array}{lll}1 & 0 & -1\end{array}\right],\left[\begin{array}{lll}1 & 1 & 0\end{array}\right]$} \\
\hline$(-1-11)$ & {$[0-1-1],\left[\begin{array}{lll}1 & 0 & 1\end{array}\right],[-1-10]$} \\
\hline$(-111)$ & {$\left[\begin{array}{lll}0 & 1 & -1\end{array}\right],\left[\begin{array}{lll}1 & 0 & 1\end{array}\right],\left[\begin{array}{lll}-1 & -1 & 0\end{array}\right]$} \\
\hline
\end{tabular}

material parameter and is independent of the lattice orientation or the considered slip system.

The discussed material with a face-centred cubic (FCC) crystal has four slip planes with three slip directions per slip plane. The FCC lattice structure with one of the twelve slip systems is shown in Fig. 5. All the corresponding slip planes and slip directions are listed in Table 1.

The material behaviour of each grain is captured by a simplified phenomenological elastoplastic constitutive law with a linear kinematic hardening. It is assumed here that the applied stress resolved along the slip direction on the slip plane (to give the shear stress) initiates and controls the extent of plastic deformation. Yield begins on a given slip system when the shear stress on this system reaches a critical value, called the critical resolved shear stress, independent of the tensile stress or any normal stress acting on the lattice plane.

For each grain $g$, the local yield criterion $f_{\mathrm{g}}\left(\sigma_{\mathrm{g}}\right)$ is obtained by the Schmid law. The individual yield stress $\sigma_{\mathrm{g}}^{\mathrm{c}}$ depends on the active slip (gliding) system $s$

$\sigma_{\mathrm{g}}^{\mathrm{c}}=\min _{s} \frac{\tau_{0}}{S_{\mathrm{g}}^{s}}$

The critical resolved shear stress $\tau_{0}$, the Young modulus $E$ and the macroscopic kinematic hardening modulus $H$ were identified from a macroscopic tensile test. The identification of the mesoscopic material parameters was done using the grain inclusion embedded in a homogeneous elastoplastic
Table 2

The macroscopic material parameters: $E$ Young modulus, $v$ Poisson ratio, $\sigma_{\mathrm{y}}$ yield limit, $\sigma_{\mathrm{Ts}}$ ultimate stress limit and $H$ kinematic hardening modulus

\begin{tabular}{lllll}
\hline$E[\mathrm{GPa}]$ & $v$ & $\sigma_{\mathrm{y}}[\mathrm{MPa}]$ & $\sigma_{\mathrm{Ts}}[\mathrm{MPa}]$ & $H[\mathrm{MPa}]$ \\
\hline 210 & 0.3 & 800 & 1300 & 4000 \\
\hline
\end{tabular}

matrix and by adjusting the parameters so as to give a response, which when averaged over all the grains corresponded to the macroscopic behaviour as illustrated in Fig. 6.

The complete set of macroscopic material parameters is presented in Table 2.

\subsection{Computation of the fatigue criteria}

All the FEM stress computations were performed using the object oriented finite element toolbox Cast3M [13] which includes a number of preprocessing, solving and postprocessing routines. Parts of the postprocessing procedures were performed using MATLAB.

The loading case discussed in this paper corresponds to five cycles of a pure plane strain bending of the specimen as described in Fig. 4.

Once the stabilized stress and strain fields are obtained, the stress results of the FEM computations have then been post processed in the following steps:

1. Determination of the shakedown state. This has been checked by computation of the relative shift of the cumulated plastic strain of the last loading cycle when compared to the one before. The shift is interpreted as plastic shakedown or ratcheting if it exceeds a limiting value and as elastic shakedown otherwise. The limiting value was determined empirically from observations of single grains.

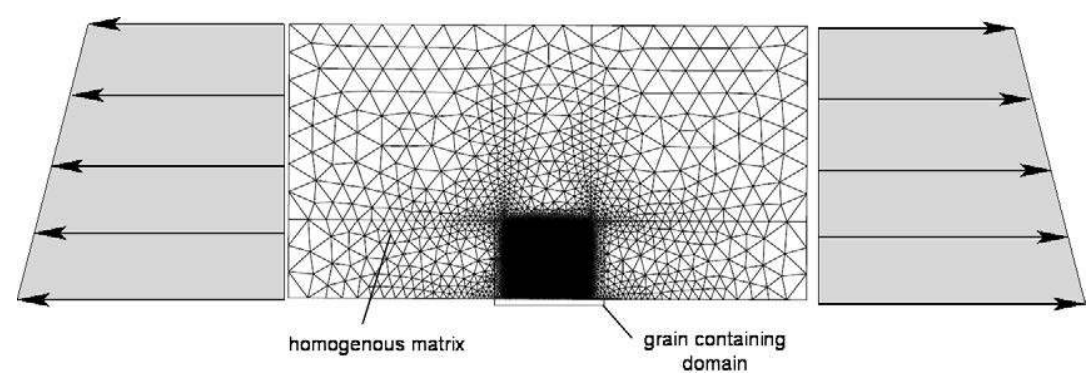

Fig. 4. Grain box embedded in the homogenous matrix and the applied boundary tractions creating a stress gradient in the specimen. 


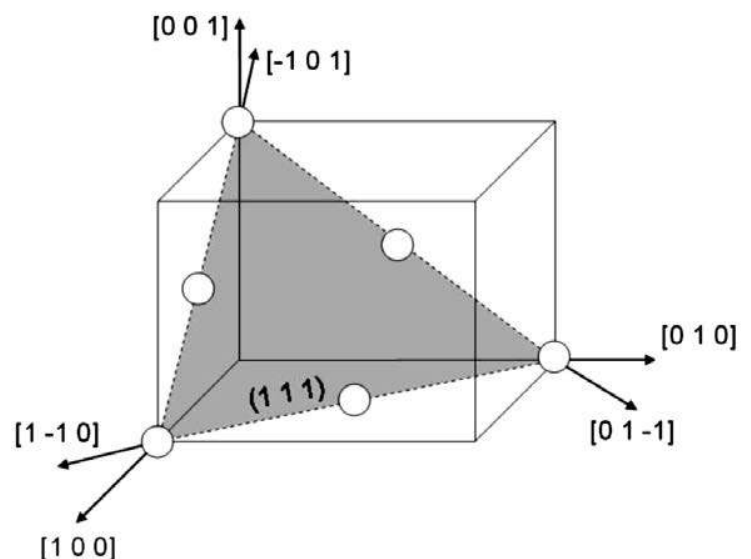

Fig. 5. FCC crystal with one slip plane and its associated slip directions.

2. Computation of the Dang Van criterion for each slip system in each grain (slip system projection) $[2,14]$. The computation of the Dang Van criterion for each slip system is done using the precise knowledge of the grain orientation and the slip systems of the grain. As such, one can precisely compute the mesoscopic shear and pressure on each slip system, and compute the inequality in [2] in each case in order to determine the critical grain and slip direction.

3. Computation of the Dang Van fatigue criterion in each grain. The computation of the criterion $[2,14]$ in each grain is done using the classical algorithms of the Dang Van criterion with the mean stress field computed over each grain as an input value.

4. Computation of the Dang Van fatigue criterion for the homogenous structure. The computation of the criterion in $[2,14]$ is done in this case by using

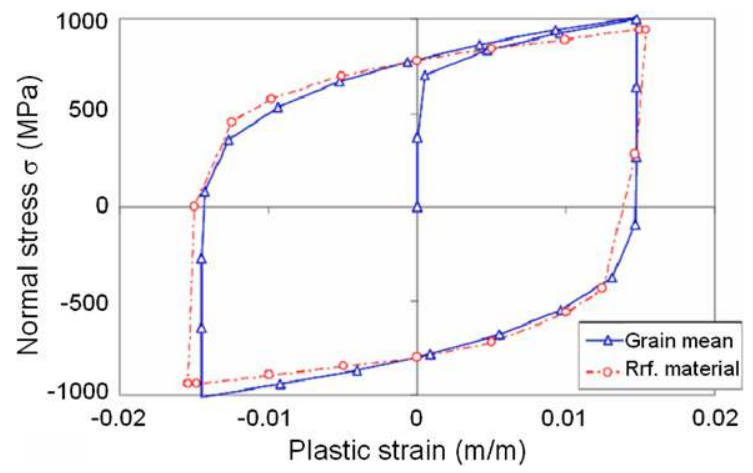

Fig. 6. Simulated grain mean and experimental behaviours of a cyclic tension test. the classical algorithms of the criterion with the stress field computed from a homogenous elastic structure submitted to the same load. In this case only the hot spot of the structure, i.e. most critical point, is plotted in the diagram [2].

\section{Results and discussion}

\subsection{Preliminary results: uniform applied load}

The first step was to confirm that the number of grains constitutes a representative sample and that shear obtained by the projection of the mesoscopic stress vector onto the slip systems leads to values similar to the mesoscopic pressure and shear obtained with the assumptions taken in the Dang Van criterion. For this purpose, the model was loaded with a fully reversed shear load. In this case,
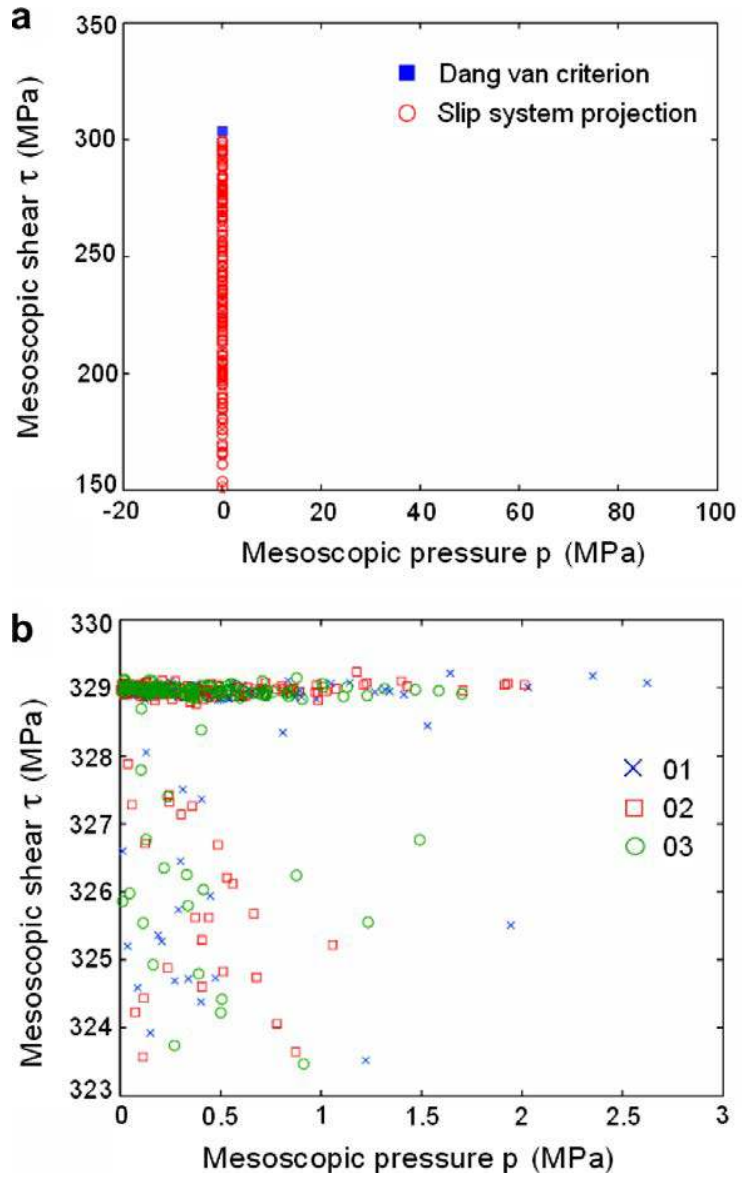

Fig. 7. Grain critical instant in the Dang Van plot: (a) case of a reversed shear loading inducing purely elastic strains; (b) case of a reversed shear loading inducing elastoplastic strains. 
the stress tensors of the macroscopic and mesoscopic stresses are equal due to the absence of hydrostatic pressure. Thus, in the case of a fully reversed loading, the centre of the smallest hypersphere circumscribing the stress path is zero in all its components. The mesoscopic shear is thus equal to the amplitude of the imposed shear stress.

Fig. 7a represents the values of mesoscopic shear against mesoscopic pressure determined with the criterion in [2] as well as with the slip system projection. The most critical point of the stress path of the last cycle is plotted for each grain. It can be seen that the points of the most critical instants determined with the criterion in [2] are represented by the same point that corresponds to the homogeneous solution.

The grain values computed with the slip system projection vary in their magnitudes of mesoscopic shear. This is a direct consequence of the different lattice orientations of the metallic grains which lead to different projections for the shear stress on the slip direction. The criterion in [2] when applied to the homogeneous solution computes the maximal mesoscopic shear under the assumption of an uniform spatial orientation of slip directions, as it will homogenise the solution over a large number of grains. Thus, under a homogeneous applied stress, both criteria provide equal points in the diagram, proving that the homogenisation over the 150 grains functioned as expected.

In a next step, the shear stress was increased to a range where some of the grains were plastified.
Fig. $7 \mathrm{~b}$ shows the values of mesoscopic shear and pressure obtained with the Dang Van criterion for this loading. It can be observed that the range of mesoscopic shear and pressure increases with the initiation of yielding in several grains. This is a direct consequence of the residual stress field induced by grain plasticity. The stress components $\sigma_{x x}$ and $\sigma_{x y}$ of the residual stress field of the unloaded specimen after the last load cycle are displayed in Fig. 8. The plot of the $\sigma_{x y}$ residual stresses gives an overview about the plastified grains. The grains are surrounded by residual stresses (see $\sigma_{x x}$ distribution) which induce the hydrostatic pressure and cause the spread of points in Fig. $7 \mathrm{~b}$.

In addition to the grain orientation (O1) observed with EBSD, two further calculations were performed with randomly (uniform distribution) generated lattice orientations $(\mathrm{O} 2, \mathrm{O} 3)$ based on the same grain structure. The clouds of grain critical instants with different lattice orientations differ insignificantly where the primary orientation $(\mathrm{O} 1)$ contains the most critical grains. That confirms that the model contains a sufficient number of grains and leads to rather conservative results.

\subsection{Influence of the stress gradient}

As in the case of the shear loading, the first load in the case of a bending moment induced a common elastic shakedown state for all the grains. The mesoscopic pressure and shear computed with the Dang Van criterion as well as with the slip system

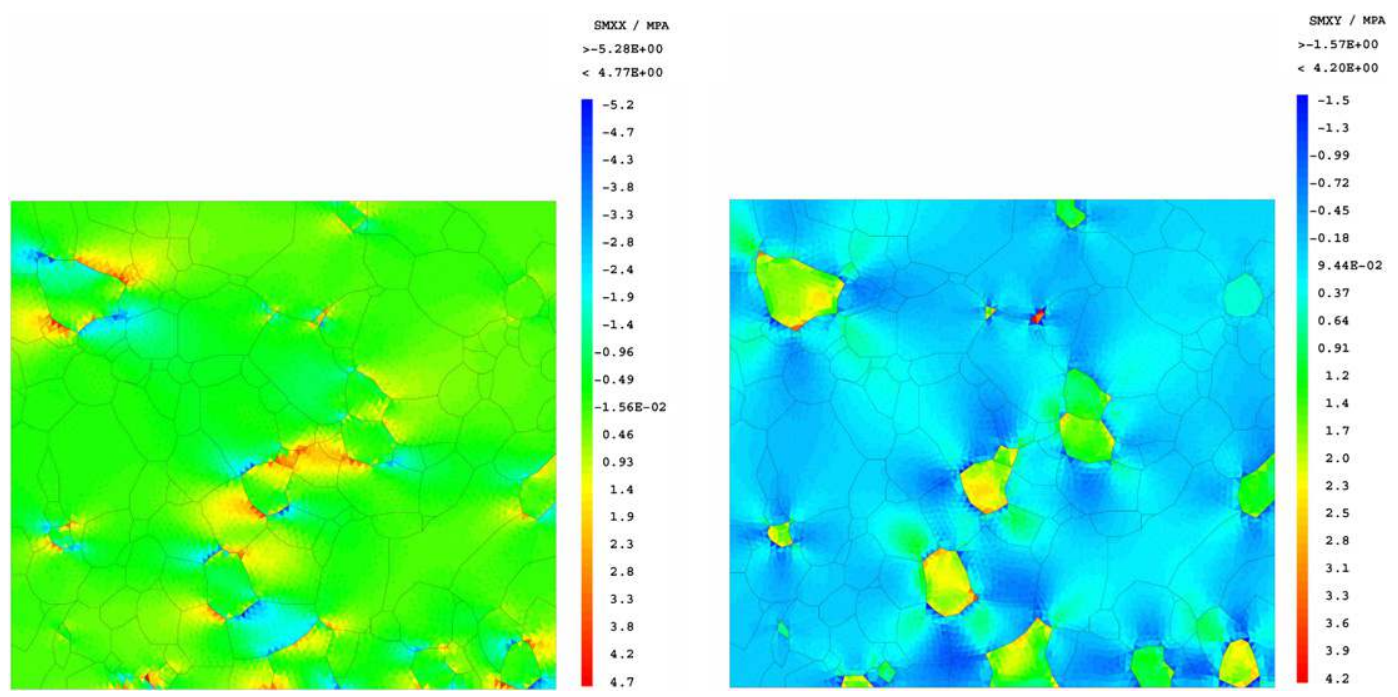

Fig. 8. $\sigma_{x x}$ and $\sigma_{x y}$ components of the residual stress field after the last loading cycle. 


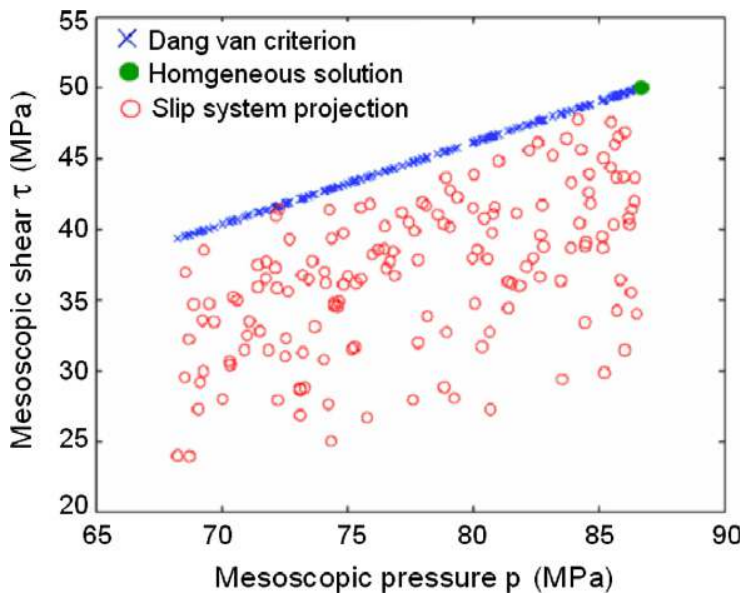

Fig. 9. Critical instant of the stress path for each grain in the Dang Van plot for a repeated bending loading.

projection are compared with the homogeneous solution in Fig. 9. One can remark that the homogeneous solution can be used as a good reference for the grain values of the computation [2] which are right below it. The points form a straight line, because the values of pressure and shear depend only on the grain's position in the grain mesh and consequently on the imposed pressure.

However, regarding the shear in the slip systems, it can be observed that the most critical grain is placed a certain distance from the point representing the homogeneous solution. Additionally, the mesoscopic shear in the slip systems of some less stressed grains is less than $50 \%$ of the value of the homogeneous solution.

As the difference between the two computations is only the apparition of a stress gradient this indicates that the stress gradient has a non-negligible effect on the fatigue limit.

A more significant influence can be observed if the imposed stress exceeds the yield limit of some grains located in the most stressed zone of the structure. From the macroscopic point of view, the material response has to be in elastic shakedown in order to permit the computation of the homogeneous solution and the application of the criterion [2]. Thus, the value of the applied load has to be between the yield limit of the weakest grain and the macroscopic elastic shakedown limit of the reference material. Fig. 10 shows the plots [2] for a load with a common maximum pressure of $700 \mathrm{MPa}$ applied with four different stress gradients. In order to improve the understanding of the diagram, only the points in the neighbourhood of the

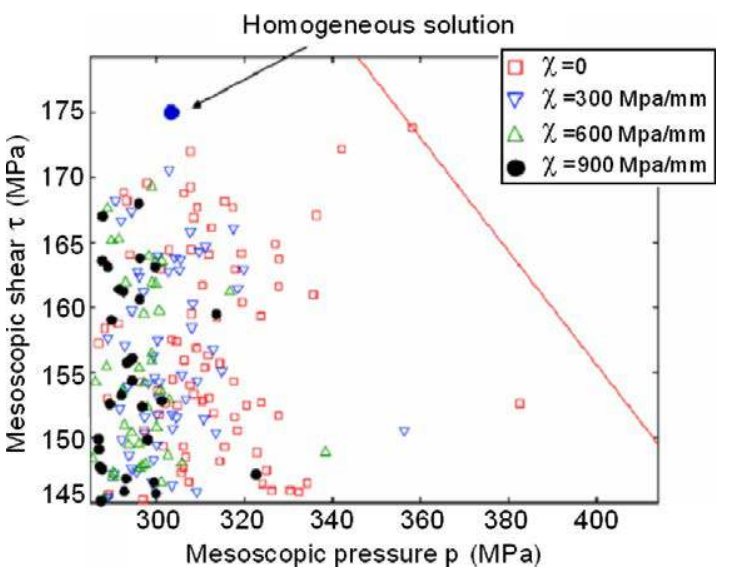

Fig. 10. Critical instant of the stress path for each grain in the Dang Van plot for different stress gradient.

most critical grain are plotted. A tentative line [2] of infinite lifetime is drawn through the point of the most critical grain belonging to the data set with a stress gradient $\chi=0$ in order to suggest a critical line [2].

The large range of mesoscopic pressure indicates the progress of plastic strain within the grains. It can be seen that the distance between the most critical grain and the line [2] increases with an increasing stress gradient. Here, it seems the distance of the clouds to the suggested line [2] is increased with increasing the stress gradient.

Let us recall that the high stress gradient will impose a varying stress level on the population of the 156 grains under observation, more precisely only about $10-15$ grains will sustain the maximal applied stress level. Moreover each of these grains has a particular orientation which implies that only a small number of slip directions will enter the computation when the fatigue criterion is projected over the slip systems. However when computing the Dang Van criterion the underlying assumption is a maximisation over all possible planes, as slip systems are supposed to be homogenously distributed. The difference displayed in Fig. 10 between slip systems and homogenous computation illustrates the fact that homogenisation does not function for a high stress gradient.

Compare the critical instants of each grain with the homogeneous solution. And regard it as an additional factor the pressure, that the macroscopic application of the criterion in [2] is more conservative than the grain values of the higher gradients, but less conservative for the lower gradients. This is a consequence of a distribution of plastic strain 
Table 3

Shakedown state of the grains for various stress gradients

\begin{tabular}{lrrrr}
\hline Stress gradient $\chi[\mathrm{MPa}]$ & 0 & 300 & 600 & 900 \\
Number of grains remaining elastic & 28 & 51 & 86 & 107 \\
Number of grains in elastic shakedown & 128 & 105 & 70 & 49 \\
Number of grains in plastic shakedown & 0 & 0 & 0 & 0 \\
Plastified area/ total area [\%] & 75 & 66 & 46 & 32 \\
\hline
\end{tabular}

in same grains which is smoothed out when higher gradients are applied.

In order to estimate the quantity of plasticity in the sample the total number of grains which are in elastic shakedown as well as the fraction of the plastified area has been analysed. The results are summarised in Table 3.

As one can see, the number of plastified grains and the plastified area decreases significantly with an increasing value of the stress gradient. Fig. 11 shows the plastic strains for two different stress gradients after the applied load cycles. One can also remark an increase of the maximum value of $\varepsilon_{x x}^{\mathrm{pl}}$ of more than $50 \%$, when the gradient is increased from $\chi=0 \mathrm{MPa} / \mathrm{mm}$ to $\chi=900 \mathrm{MPa} / \mathrm{mm}$.

In the previous paragraph, an important influence of the stress gradient has been observed, as displayed using the projections on the slip directions in each grain in the plots [2]. However, up to now, the identification of the material parameters $a$ and $b$, parameters defining the fatigue limit, has not been discussed. The fatigue life line is defined according to theory by the elastic shakedown limit at the grains scale. Use the preceding computations in order to determine precisely the shakedown limit at the grain scale and define in this way the fatigue limit. The natural question underlying to this procedure is: does the fatigue limit depend of the macroscopically applied stress gradient?

\subsection{Shakedown and fatigue limit with stress gradient}

Thus, the fatigue limit was determined following the steps of the technique described below:

1. Apply a macroscopic load with the given stress gradient in order to attain only the yield limit of the weakest grain.

2. Compute the projection on each slip system for all grains.

3. Determine the shakedown state of each grain.

4. If all grains are in elastic shakedown, increase the apply load with fixed increment, and continue with step 2 until to reach a plastic shakedown.

This procedure for identifying the fatigue limit, considered as an elastic shakedown threshold was performed for five constant stress gradients $\chi$. The results of these fatigue simulations are presented in Fig. 12a.

In order to interpret the results, let us recall that the simulated fatigue tests discussed before were all performed with a repeated load where $\sigma_{\min }=0$. In the computational approximation to the fatigue limit, only the maximum stress was varied. Regarding the different mean values in the calculated fatigue limits, there is a mean stress effect besides the stress gradient effect which has to be considered in

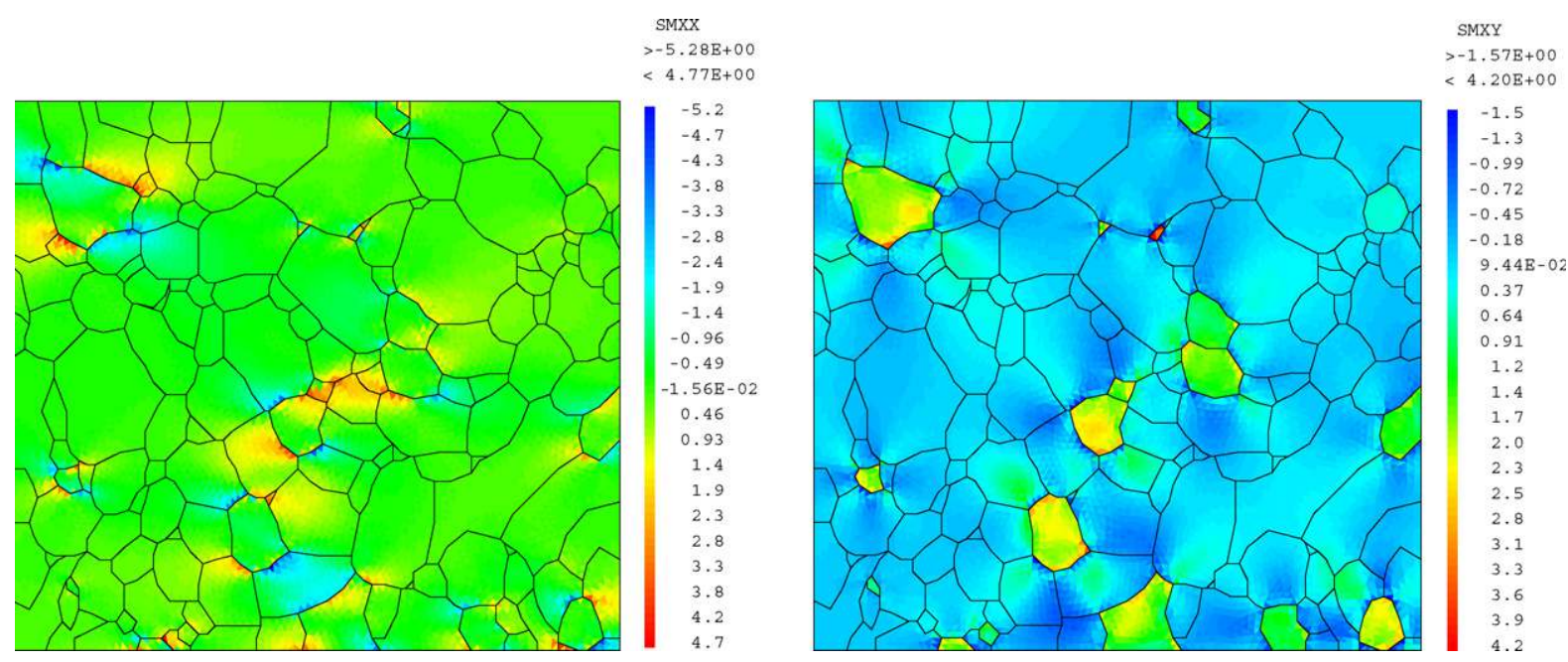

Fig. 11. Plastic mesostrain for $\chi=0$ and $\chi=900 \mathrm{MPa} / \mathrm{mm}$ for a common maximum stress of $\sigma_{x x}=700 \mathrm{MPa}$. 

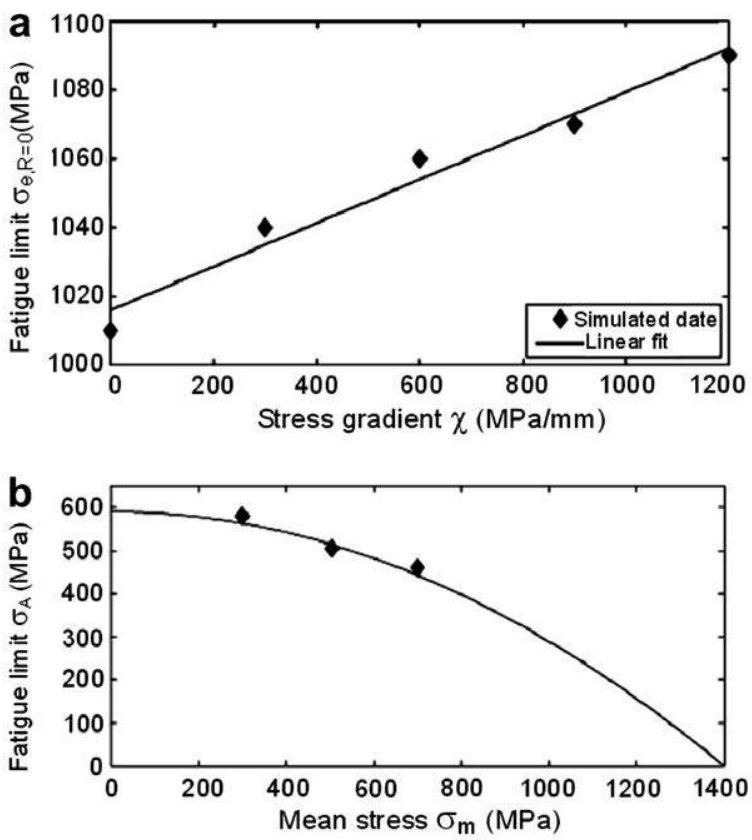

Fig. 12. Fatigue limit results: (a) computed fatigue limits as a function of the stress gradient; (b) computed fatigue limits for different mean stresses.

the results presented before. This mean stress influence was analysed with further fatigue simulations of repeated loadings with different mean stresses.

This influence can be simply quantified in terms of the law of Gerber parabola:

$\sigma_{\mathrm{A}}=\left.\sigma_{\mathrm{e}}\right|_{\sigma_{\mathrm{m}}=0}\left[1-\left(\frac{\sigma_{\mathrm{m}}}{\sigma_{\mathrm{TS}}}\right)^{2}\right]$

where $\left.\sigma_{\mathrm{e}}\right|_{\sigma_{\mathrm{m}}=0}$ is the endurance limit for a fully reversed loading $(R=-1)$ and $\sigma_{\mathrm{TS}}$ is the ultimate tensile strength. This assumption was taken in accordance with its experimental validation in uniaxial loading of ductile materials like it has been the case in the simulations. The endurance limit in reversed tension-compression $\left.\sigma_{\mathrm{e}}\right|_{\sigma_{\mathrm{m}}=0}$ as well as the tensile strength $\sigma_{\mathrm{TS}}$ in Eq. (7) were considered as "scale parameters" to achieve the best fit for the computed values. Therefore, two further fatigue limits in tension-compression with different mean stresses were computed such that three pairs of values (see Fig. 12b) were at the disposal to obtain the values of $\left.\sigma_{\mathrm{e}}\right|_{\sigma_{\mathrm{m}}=0}$ and $\sigma_{\mathrm{TS}}$ which are, respectively, equal to $590 \mathrm{MPa}$ and $1400 \mathrm{MPa}$. It is interesting to remark that the obtained values match the material parameters of the referenced steel $42 \mathrm{CrMo} 4$,

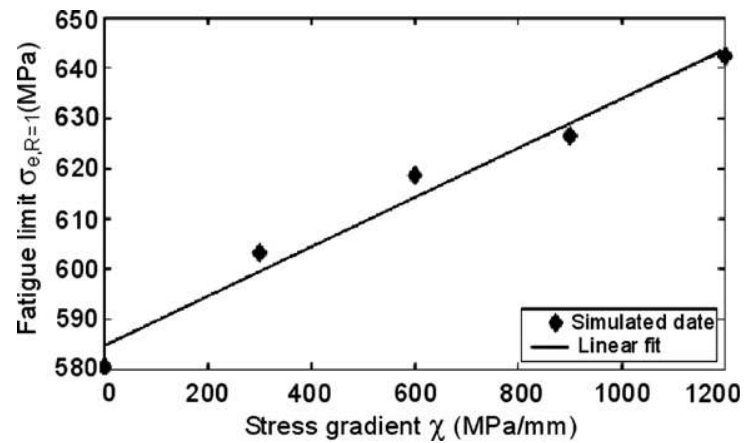

Fig. 13. Computed fatigue limits related to the common mean stress $\sigma_{\mathrm{m}}=0$.

which has a material behaviour close to the one represented in Fig. 3.

With the model specific parameter $\sigma_{\mathrm{TS}}$, the stress gradient dependent fatigue limits can be related to a common mean stress. In order to compare the computed data with experimental results, this mean stress was chosen to $\sigma_{\mathrm{m}}=0$ (reversed loading). The $\sigma_{\mathrm{e}}-\chi$ plot of the related values is shown in Fig. 13.

The linear trend is in accordance with the experimental fatigue results obtained on cylindrical rotating bending specimens with various radii in [16].

\subsection{Adaptation of the Dang Van criterion}

The computed shakedown limits was used to determine the parameters $a$ and $b$ of the fatigue criterion in [2].

The material parameter $b$ equals the fatigue limit on reversed torsion loading. Accepting the experimentally justified fact that the fatigue limit for shear loads is not affected by a stress gradient effect [16], the parameter $b$ was fixed and only the slope of the line [2] of infinite lifetime was varied.

However, obtaining the fatigue limit with the shakedown criterion for alternating shear load was less simple. All the grains responded with a plastic shakedown state if the equivalent stress exceeded the local yield limit of the grains. A possible explanation is that the number of imposed load cycles was not sufficiently large to impose the elastic shakedown state. Considering the experimental results of $[15,17]$, the fatigue limit for alternating shear was considered to be the shear yield limit of the weakest grain. As a consequence the fatigue limits for alternating shear is given by the following relation: 


$$
\begin{aligned}
\tau_{\mathrm{e},-1}^{\mathrm{T}} & =\min _{\mathrm{gr}} \tau_{\mathrm{y}}^{\mathrm{gr}}=\min _{\mathrm{gr}}\left(\frac{\sigma_{\mathrm{y}}^{\mathrm{gr}}}{\sqrt{3}}\right)=\frac{1}{\sqrt{3}} \frac{\tau_{0}}{S_{\max }} \\
& =323 \mathrm{MPa}
\end{aligned}
$$

The slope $a$ of the infinite lifetime line [2] can be achieved in two different ways:

(a) Using the fatigue limits of a reversed bending associated with the shakedown criterion. This technique is not accurate, as the values because the alternate fatigue limits are from an empiric Gerber law.

(b) By drawing the line through the point $[0, b]$ and the most critical instant of the stress path of (i) the homogeneous solution, (ii) the most critical grain in plastic shakedown or (iii) the most stressed grain regarding to the values of mesoscopic shear and pressure.

Taking into account the expected accuracy, procedure (b) can be tested. The results based on the threes different points for stress distributions with $\chi=300 \mathrm{MPa} / \mathrm{mm}$ and $\chi=1200 \mathrm{MPa} / \mathrm{mm}$ are displayed in the Dang Van diagram shown in Figs. 14 and 15. It shows the most critical instant of the mesoscopic stress path for every grain. As one would expect, the line defined by the grain with the maximum values of mesoscopic shear and pressure has the smallest slope and represents consequently the most conservative approach. One can generally remark that the cloud of values computed using the criterion [2] in each grain is largely spreaded and does not permit to draw ay conclusion. However, the projection over the slip systems in each grain are a consistent approach and are generally well placed under the line defined by the

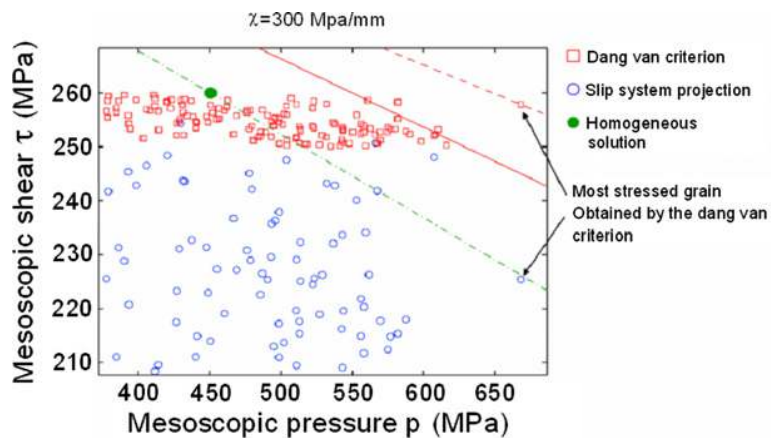

Fig. 14. Grain critical instants at the computed fatigue limit for $\chi=3 \mathrm{MPa} / \mathrm{mm}$ and included lines of infinite lifetime in the Dang Van plot.

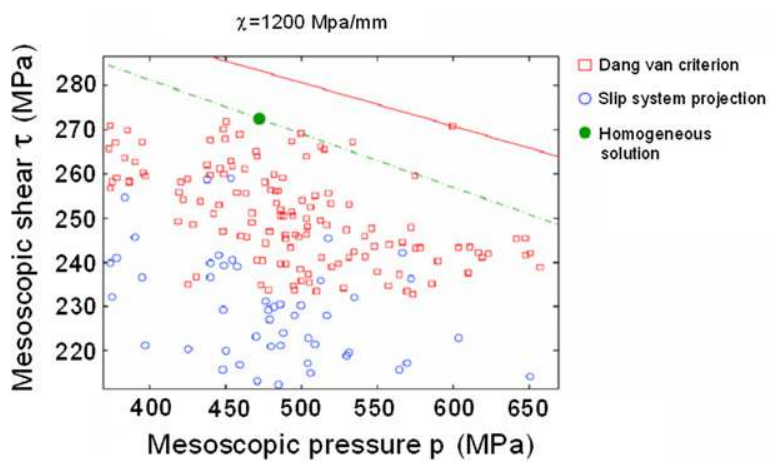

Fig. 15. Grain critical instants at the computed fatigue limit for $\chi=12 \mathrm{MPa} / \mathrm{mm}$ and included lines of infinite lifetime in the Dang Van plot.

homogeneous computation as one would expect. Moreover the distance between the homogeneous solution and the cloud of projection on the slip system is increased with increasing stress gradient if the maximal stress is constant. This is in accordance with the fact that the mean stress over all grains will decrease with an increasing gradient.

\section{Conclusions}

This work presented a numerical fatigue analysis of specimens using a simplified polycrystalline model at the mesoscopic/grain scale. The impact of the applied stress gradient on fatigue was shown considering the example of a bending experiment.

It was shown that high localization of stresses causes the failure of the homogenisation assumptions as only few grains are exposed to very high stresses. This failure implies a fundamental change in the application of fatigue criteria in general and more particularly of critical plane criterion [2] in cases of high stress gradient. The results also provide a physical explanation for critical distances and notch factors encountered in engineering practice.

A finer quantitative analysis both at the mesoand macroscopic level should allow the development of a better understanding and ability to predict fatigue for stress states involving high gradients such as those found in notched or cracked specimens and bending experiments.

On the one hand side, a direct measurement of surface strains is actually possible using optical strain analysis under electronic microscopy could be used to check a certain number of assumptions of the presented work. 
On the other hand side, the numerical modelling itself can be also improved by using a more complex hardening model in the polycrystalline behaviour. It is also obvious that finer structures like surface state, grain boundaries, inclusions and impurities have been completely neglected in this frame setting.

A further drawback is the usage of a simple two dimensional computation under a plane strain assumption. The three dimensional computation does not address a more complex material behaviour, but demands a three dimensional measurement of the grain structure which was not available at the time of the work. However this could be obtained sometime in the near future.

\section{References}

[1] K. Dang Van, I.V. Papadopoulos, High-cycle metal fatigue, from theory to applications, in: K. Dang Van, I.V. Papadopoulos (Eds.), CISM Courses and Lectures no. 392, Springer-Verlag, 1999.

[2] K. Dang Van, A high cycle fatigue criterion applied in biaxial and triaxial out-of-phase stress conditions, in: D.L. McDowell, R. Ellis (Eds.), Advances in Multiaxial Fatigue, ASTM STP, 1191, ASTM, Philadelphia, 1993, pp. 120-130.

[3] R.A. Naik, D.B. Lanning, T. Nicholas, A.R. Kallmeyer, A critical plane gradient approach for the prediction of notched HCF life, Int. J. Fatigue 27 (5) (2005) 481-492.

[4] D. Taylor, Geometrical effects in fatigue: a unified theoretical model, Int. J. Fatigue (21) (1999) 413-420.

[5] H. Adib, G. Pluvinage, Theoretical and numerical aspects of the volumetric approach for the fatigue life for the notched components, Int. J. Fatigue (25) (2003) 67-76.
[6] A. Banvillet, T. Palin-Luc, S. Lasserre, A volumetric energy based high cycle multiaxial fatigue criterion, Int. J. Fatigue 25 (8) (2003) 755-769.

[7] F. Morel, T. Palin-Luc, C. Froustey, Comparative study and link between mesoscopic and energetic approaches in high cycle multiaxial fatigue, Int. J. Fatigue 4 (23) (2001) 317-327.

[8] F. Morel, T. Palin-Luc, A non-local theory applied to highcycle multiaxial fatigue, Fat. Fract. Eng. Mat. Struct. 25 (7) (2002) 649-665.

[9] D. Nowell, D. Dini, Stress gradient effects in fretting fatigue, Tribol. Int. 36 (2) (2003) 71-78.

[10] S. Suresh, Fatigue of Materials, second ed., Cambridge University Press, Cambridge UK, 1998.

[11] G.J. Weng, A Micromechanical theory of grain-size dependence in metal plasticity, J. Mech. Phys. Solids 31 (3) (1983) 193-203.

[12] K. Kowalczyk, W. Gambin, Model of plastic anisotropy evolution with texture-dependent yield surface, Int. J. Plast. 20 (2004) 19-54.

[13] CAST3M.: An object oriented finite element toolbox, http:// www-cast3m.cea.fr/.

[14] I.V. Papadopoulos, A new criterion of fatigue strength for out-of phase bending and torsion of hard metals, Int. J. Fatigue 16 (6) (1994) 377-384.

[15] R.G. Pogoretskii, G.V. Karpenko, Effect of test piece length on the fatigue strength of steel in air, Proc. Khimicheskaya Mekhanika Materialov 1 (1965) 90-94.

[16] I.V. Papadopoulos, Invariant formulation of a gradient dependent multiaxial high-cycle fatigue criterion, Eng. Fract. Mech. 55 (1996) 513-528.

[17] G. Sines, Behavior of metals under complex static and alternating stresses, in: S. Waisman (Ed.), Metal Fatigue, McGraw-Hill Book Company, New-York, 1959, pp. 145169. 